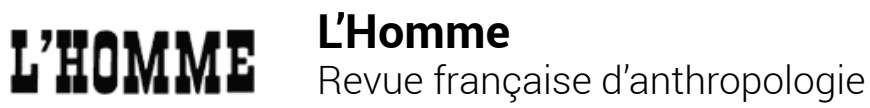

187-188 | 2008

Miroirs transatlantiques

\section{Pierre-Jean Simon, Pour une sociologie des relations interethniques et des minorités}

\section{Thomas Pierre}

\section{(2) OpenEdition \\ 9 Journals}

\section{Édition électronique}

URL : https://journals.openedition.org/lhomme/20632

DOI : 10.4000/lhomme.20632

ISSN : 1953-8103

Éditeur

Éditions de l'EHESS

\section{Édition imprimée}

Date de publication : 3 octobre 2008

Pagination : 484-486

ISBN : 978-2-7132-2186-6

ISSN : 0439-4216

Référence électronique

Thomas Pierre, "Pierre-Jean Simon, Pour une sociologie des relations interethniques et des minorités », L'Homme [En ligne], 187-188 | 2008, mis en ligne le 16 décembre 2008, consulté le 23 avril 2022. URL http://journals.openedition.org//homme/20632 ; DOI : https://doi.org/10.4000//homme.20632

Ce document a été généré automatiquement le 23 avril 2022.

(c) École des hautes études en sciences sociales 


\title{
Pierre-Jean Simon, Pour une sociologie des relations interethniques et des minorités
}

\author{
Thomas Pierre
}

\section{RÉFÉRENCE}

Pierre-Jean SIMON, Pour une sociologie des relations interethniques et des minorités. Rennes, Presses universitaires de Rennes, 2006, 347 p., notes bibliogr., fig.

1 CET OUVRAGE réunit plusieurs contributions de Pierre-Jean Simon. L'ensemble relève du champ de ce qu'il est convenu d'appeler la sociologie des relations interethniques, constituée des "relations raciales", des relations interculturelles, des problèmes de minorités, de l'immigration, ainsi que des questions régionale, nationalitaire et nationale.

D'emblée, l'auteur postule que ce domaine a longtemps été négligé en France. Il explique cette spécificité nationale - qualifiée «d'idiosyncrasie française »- par l'histoire complexe des sciences sociales dans ce pays. Selon lui, l'analyse par les sciences sociales des relations interethniques ne peut plus - comme cela a été longtemps le cas du fait de très fortes résistances idéologiques au nom d'une certaine «tradition républicaine » - être négligée. Ainsi l'ouvrage se veut-il une contribution à la constitution de ce champ de recherche, lequel est à situer dans le cadre théorique de la sociologie transversale de la différenciation et de la hiérarchisation sociales. Une attention particulière est accordée aux obstacles idéologiques dont doit tenir compte toute démarche scientifique s'inscrivant dans ce champ.

Dans son premier chapitre, Pierre-Jean Simon insiste sur l'exigence de précision et de clarification que suppose toute démarche d'analyse sociologique. Les mots étant des artefacts au sens propre. Un mot n'ayant pas de sens mais des emplois. Cette exigence est d'autant plus indispensable quand ces mots abstraits - tels que la race et le racisme 
ou la nation et la nationalité - sont chargés de connotations idéologiques, et qu'ils constituent parallèlement des pièces importantes dans les systèmes idéologiques en compétition politique passée et/ou contemporaine. En ce sens, la rigueur dans les définitions des concepts se pose à la fois comme une exigence de méthode mais aussi comme une question de principe. En effet, quand le signifiant devient n'importe quoi, le signifié perd toute réalité. Pour illustrer son propos, l'auteur s'attarde sur la banalisation de l'usage du terme "fasciste » avant de souligner que, dans le domaine social et politique, manipuler les mots revient à manipuler les représentations.

Cet appel à l'examen critique préalable se justifie par le fait que la vérité scientifique se trouve dans les abstractions conceptuelles. Ce sont les concepts qui permettent de questionner la réalité. L'auteur s'appuie alors sur les postures respectives d'Émile Durkheim et de Max Weber. Il voit dans chacune d'entre elles un souci quasi obsessionnel de la terminologie; une leçon essentielle pour qui tente de rendre intelligible le monde social. Aussi, l'analyse critique des mots - fondée sur l'histoire sociale de leur genèse, de leurs emplois et de leurs différentes utilisations - est considérée comme un préalable indispensable. C'est pourquoi, l'auteur appelle au nominalisme méthodologique.

Dans le deuxième chapitre, il est question d'épistémologie, du lien entre idéologie et sociologie. Quels rapports entretient la sociologie des relations interethniques aux idéologies ? Pour l'auteur, la prétention à la parfaite objectivité et neutralité dans les sciences sociales est toujours un leurre. Mais, si la constitution d'un savoir scientifique, parce qu'elle rencontre beaucoup d'obstacles, est forcément lente quand elle traite de questions où l'idéologisation des analyses sociologiques est particulièrement importante, ces analyses permettent pour le moins de poser plus clairement que partout ailleurs - dans la mesure où l'on ne peut ici le contourner - le problème de l'idéologie. Pour Pierre-Jean Simon, toute réflexion sur l'idéologie se réfère en fait à la conception de Marx. En effet, s'il n'a pas créé le mot, Marx a réellement inventé le concept d'idéologie. Pour Marx, les idéologies signifient les idées fausses que les hommes se font d'eux-mêmes. Les représentations irréelles, imaginaires, fantasmatiques de leurs rapports au monde et entre eux. L'idéologie demeure le propre de la conscience aliénée, rendue étrangère à elle-même et déréalisée.

6 L'auteur fait un constat central: les idéologies, éléments consubstantiels de la modernité, tout comme les sciences, partagent avec elles les mêmes postulats fondamentaux, liées au même processus historique de rationalisation et d'intellectualisation; en cela, elles se posent en concurrentes directes des sciences et particulièrement des sciences sociales. Mais comment peut-on distinguer la science de l'idéologie ? Pour l'auteur, le critère pertinent de distinction réside dans la faillibilité. La démarche scientifique se sait faillible. Elle admet l'idée qu'elle n'est que temporaire, provisoire, discutable et réfutable. Le savoir scientifique est constitué d'une série d'erreurs peu à peu rectifiées, toujours de manière approximative. C'est en cela qu'une discipline à projet scientifique est susceptible de progrès cumulatif. La sociologie n'a pu naître historiquement qu'avec l'apparition du doute social. Il reste que la critique sociologique des idéologies ne vise pas à la démolition de tout système d'idées et de valeurs mais à la prise de conscience et à la mise en évidence de leur non-appartenance à la sphère de la connaissance de type scientifique.

7 Dans son troisième chapitre, Pierre-Jean Simon insiste sur le fait que le cadre théorique général de référence au sein duquel l'analyse des relations interethniques doit, à son 
sens, se situer - à savoir la sociologie transversale de la différenciation et de la hiérarchisation sociales - permet de refuser toute pensée providentialiste et naturaliste pour ne considérer que les déterminants sociaux inégalitaires établis entre les êtres humains. L'ordre des classements sociaux - selon l'âge, le sexe, l'origine et l'appartenance ethnique ou nationale, et la catégorie socioprofessionnelle - ne trouve en aucun cas ses principes en dehors du social. Les rapports entre les différents ensembles collectifs classés suivant ces quatre grands modes de la différenciation et de la hiérarchisation sociales amènent à saisir ceux-ci dans une même problématique, dans le cadre d'une théorie générale des classements sociaux, théorie interprétative permettant une lecture cohérente de la différenciation et de la hiérarchisation sociales. Cette posture transversale permet de combiner entre eux les différents modes de classements sociaux.

8 Puis, l'auteur s'attarde sur les divers sens et usages du terme " minorité » avant de se consacrer à la situation minoritaire. Ce qui apparaît central dans le concept sociologique de minorité, c'est la minorisation, la situation de domination, de dépendance et d'exclusion. C'est généralement l'idée d'incapacité - temporaire ou définitive, selon que l'on se place dans des perspectives évolutionnistes, progressistes ou essentialistes - qui est utilisée dans les idéologies de justification de la domination par les majoritaires des minorités raciales, ethniques, régionales ou nationales. Le concept de minorité induit en lui-même une carence.

9 Par la suite, Pierre-Jean Simon explicite les trois termes communément associés au concept de minorité à savoir, la race, la culture et la nation. Il y a une grande difficulté à donner un sens sociologique à la minorité. C'est en cela que son objectivation passe par son adjectivation autour de ces trois grandes idées qui sont idéologiquement très marquées mais aussi très floues. Dans ce contexte, l'auteur consacre un chapitre à l'analyse d'un mythe, celui de la race, avant de s'intéresser à l'histoire du racisme en tant que mot et pratique.

Par ailleurs, à partir du concept d'ethnocentrisme, central dans l'étude des relations interethniques, la dernière contribution propose un schéma d'analyse des attitudes, pratiques et politiques relationnelles. Pierre-Jean Simon distingue alors le racial de l'ethnique, considérant ici l'ethnique selon l'usage traditionnel de la sociologie et de l'anthropologie nord-américaine, à savoir, l'ethnique perçu comme relevant strictement de l'ordre du socio-historico-culturel. À l'époque moderne, cet ethnocentrisme prend principalement la forme du patriotisme, le sentiment de loyauté à l'égard du groupe auquel on appartient ayant été transféré des communautés traditionnelles à la collectivité politique dont on est sujet citoyen. L'ethnocentrisme, c'est la difficulté à se penser comme existant parmi d'autres. Mais ce sentiment apparaît, selon l'auteur, comme constitutif de toute collectivité ethnique en tant que telle. En cela, l'ethnocentrisme serait un phénomène social pleinement normal. La perte de tout ethnocentrisme conduit, individuellement et collectivement, à l'assimilation par adoption de la culture d'une communauté considérée comme supérieure. C'est la question du lien entre ethnocentrisme et pérennité d'un groupe qui est ici posée. L'auteur rappelle que lorsque l'on pose cette question, il faut veiller à conserver au terme d'ethnocentrisme sa valeur de concept sociologique. En cela, toutes les attitudes ethnocentristes ne sont pas des attitudes racistes.

11 Enfin, en matière de relations inter-ethniques, Pierre-Jean Simon détermine quatre orientations fondamentales typiques des attitudes, des pratiques et des politiques: le 
racisme, l'ethnisme, l'assimilationnisme et le pluralisme. C'est par rapport au fait premier de l'ethnocentrisme que celles-ci se définissent et se distinguent. L'auteur qualifie alors le racisme d'ethnocentrisme absolutisé, l'ethnisme d'ethnocentrisme exacerbé, l'assimilationnisme progressiste d'ethnocentrisme sublimé, et le pluralisme d'ethnocentrisme délibérément contrarié.

12 Cet ouvrage tire sa pertinence de la manière dont Pierre-Jean Simon parvient à s'extraire des concepts pour les rendre plus clairs, pour en faire des référents scientifiques, en revenant sans cesse sur leur constitution historique et l'évolution sémantique de leurs usages. En cela, l'ensemble constitue clairement une proposition méthodologique exigeante et nécessaire puisque concernant un champ toujours plus complexe et potentiellement très conflictuel. 